"This is the peer reviewed version of the following article: Sánchez-Gutiérrez, C. A., RuizMéndez, M. V., Jiménez-Castellanos, M. R. and Lucero, M. J. (2017), Influence of refining processes on content of bioactive compounds, rheology, and texture of olive pomace oil for use in topical formulations. Eur. J. Lipid Sci. Technol., 119: n/a, 1600408, which has been published in final form at http://doi.org/ 10.1002/ejlt.201600408. This article may be used for non-commercial purposes in accordance with Wiley Terms and Conditions for Self-Archiving."

Influence of refining processes on content of bioactive compounds, rheology, and texture of olive pomace oil for use in topical formulations

C. A. Sánchez-Gutiérrez ${ }^{\mathrm{a}, \mathrm{b}}$, M. V. Ruiz-Méndez ${ }^{\mathrm{b}^{*}}, \mathbf{M}$. R. Jiménez-Castellanos ${ }^{\mathrm{a},{ }^{*}}$ and $\mathbf{M}$. J.

\title{
Lucero $^{\mathrm{a}, *}$
}

${ }^{a}$ Departamento de Farmacia y Tecnología Farmacéutica. Facultad de Farmacia. Universidad de Sevilla, Spain.

${ }^{\mathrm{b}}$ Instituto de la Grasa. Consejo Superior de Investigaciones Científicas, Sevilla, Spain.

*Corresponding author: IMPORTANT: The three Professors must present the asterisk for research purposes:

Prof. M. J. Lucero*, Prof. M. R. Jiménez-Castellanos*,

Dpto. de Farmacia y Tecnología Farmacéutica.

Facultad de Farmacia.

Email:mjlucero@us.es

Email:mrosa@us.es

\section{M.V. Ruiz-Méndez*} Instituto de la Grasa. Consejo Superior de Investigaciones Científicas, Sevilla, Spain. Email:mvruiz@ig.csic.es

E-mail addresses: csanchez17@us.es (C. A. Sánchez-Gutiérrez), mvruiz@ig.csic.es (M.V. Ruiz- 
31 Mendez), mrosa@us.es (M. R. Jiménez-Castellanos), mjlucero@us.es (M. J. Lucero)

32 
34 Retaining the bioactive ingredients of olive pomace oil is a crucial step in ensuring their functional or pharmaceutical value. To help select the best method for retaining bioactive compounds in olive pomace oil, three refining processes, namely, chemical, classical physical, or molecular distillation, were compared at different temperatures. For each method, the contents of triacylglycerols, fatty acids (oleic acid, linoleic and linolenic acids), triterpenic acids (oleanolic and maslinic acids), and unsaponifiable matter (squalene, aliphatic, sterols and terpenic) were studied. We observed that physical refining by molecular distillation provided oils with interesting amounts of bioactive compounds, especially triterpenic acids. Of these oils, the samples submitted to a temperature of $190^{\circ} \mathrm{C}$ exhibited a greater albeit low acidity compared with the other oils obtained by chemical and classical physical refining but exhibited higher amounts of all bioactive compounds. Conversely, the molecular distillation refining process had only a slight effect on the values of the consistency index. The firmness, cohesiveness, and adhesiveness parameters from texture profile analysis indicated that in general, all deodorized oils had high smoothness and spreadability but low adhesiveness. Molecular distillation was concluded to be the best refining process. Nevertheless, it is necessary to improve the working conditions of this process. 


\section{INTRODUCTION}

Olives are subjected to a mechanical extraction process in order to separate the different parts that make up the fruit and obtain the pomace (solid residue), extra virgin olive oil (EVOO juice), and vegetable water. EVOO has been used since ancient times as a natural product for skin-care formulations and cosmetics [1]. However, olive pomace oil (OPOC), obtained from pomace, has always been discarded or used in other technological fields. Nevertheless, in recent years, there has been growing interest in OPOC due to its composition because it contains all the functional compounds of EVOO but with a higher concentration of some minor components [2]. Thus, in decreasing order of concentration, the bioactive compounds are: a) triacylglycerols as the main component (>95\%); b) fatty acids, such as oleic acid, used in cosmetic and pharmaceutical formulation, and linoleic and linolenic acids, which influence the metabolic processes of the skin and promote ceramide formation [3] and the activity of vitamins $A$ and $E[4] ;$ c) triterpenic acids, in particular oleanolic and maslinic acids, with antitumor, anti-inflammatory, and bactericidal activities [5]; and d) unsaponifiable matter composed of squalene, used as an emollient to keep skin soft and elastic. Additionally, it has antioxidant properties due to its isoprene structure [6]. Aliphatic alcohols suppress the release of various inflammatory mediators [7]; sterols and terpenic alcohols (erythrodiol and uvaol) have positive effects on the inflammatory process [8].

Chemical and mechanical extractions can be used to obtain OPOC. In a previous paper [9], it was demonstrated that mechanical extraction provides a greater percentage of the main compounds (triacylglycerols). However, due to the high acidity of the OPOC, it cannot be used in pharmaceuticals. This is why the OPOC must be subjected to a refining process, but with minimum losses of bioactive compounds [10].

There are two refining procedures, chemical $(C)$ or physical $(P)$, with different steps. The common method of refining oil is by reacting it with an alkali solution, which neutralizes the free fatty acids (chemical refining). Ruiz-Mendez et al. [11] demonstrated that physical procedures result in minimal losses of bioactive compounds, such as triterpenic acids in the case of OPOC. In physical refining, vegetable oils can be neutralized by free fatty acid distillation at high temperatures, low pressures, and with steam stream. However, physical refining can only be applied after mechanical extraction. This is because the OPOC from chemical extraction has a high content of chlorophyll, phosphatides, carotenes, and other pollutants that cannot be removed by simple physical means [12].

Given the above, the aim of this work was to study the influence of refining processes in determining the composition of bioactive compounds for use in topical formulations. For 
quantitative evaluation of the composition, two types of OPOC were selected, obtained by chemical or mechanical extraction. These oils were subjected to various refining processes (chemical refining, classical physical refining, or physical refining by molecular distillation at different temperatures). The best refining process was selected on the basis of the analytical determination of bioactive compounds by gas chromatography. Finally, rheological characterization and texture profile analysis are discussed as convenient methods for mechanical characterization of topical olive pomace oils.

\section{MATERIALS AND METHODS}

\subsection{Materials}

The raw materials used for this paper were OPOC from the 2012-2013 campaign and were labeled as follows: olive pomace oil (OPOC) obtained by chemical extraction (OPOC C) and, two OPOCs obtained by mechanical extraction (OPOC MA and OPOC MB). All OPOCs were refined at the laboratory scale. Therefore, OPOC $\mathrm{C}$ and OPOC MA were subjected to a chemical refining process, and the obtained products were labeled as OPOD CC and OPOD MAC, respectively. On the other hand, OPOC MA and OPOC MB were refined by classical physical refining and physical refining by molecular distillation at different temperatures, and the obtained products were labeled as OPOD MAP and OPOD MBP, respectively (Fig. 1).

Different reagents and solvents were used: hexane, diethyl ether, isopropyl ether, ethanol, chloroform, dichloromethane phosphoric acid, methanol, pyridine, acetone, acetic acid, sulfuric acid, potassium hydroxide, sodium methylate, sodium hydroxide, ethyl acetate, phenolphthalein, and HPLC grade acetonitrile were purchased from Panreac SA (Barcelona, Spain). Trimethyl chlorosilane, hexamethyl disiloxane, and Sudan I were purchased from Sigma-Aldrich (Darmstadt, Germany). Filter aids were supplied by Dicalite (Gent, Belgium). Tonsil supreme 114 FF was obtained from Süd-Chemie (Toledo, Spain). All reagents and solvents used were of analytical quality.

As standard compounds, 5- $\alpha$-cholestan-3-ol, n-heicosanol, betulinic acid, lauryl arachidate, and methyl heptadecanoate were purchased from Sigma-Aldrich SA (S. Louis, USA). Squalene was purchased from Merck (Darmstadt, Germany).

\subsection{Methods}

\subsubsection{Chemical refining process}


The steps of this method are described below:

Degumming: Five hundred grams of OPOC C or OPOC MA was weighed into a vessel, and 0.3 wt.\% oil of phosphoric acid was added. The mixture was maintained at $25^{\circ} \mathrm{C}$ for $30 \mathrm{~min}$ under agitation, to precipitate phospholipids in the acidic media.

Neutralization: Without separating the gums, the required amount of $\mathrm{NaOH}\left(24^{\circ} \mathrm{Be}\right)$ was added, accompanied by an excess of $10 \%$. The addition was performed at $40^{\circ} \mathrm{C}$ and the temperature was then increased to $80^{\circ} \mathrm{C}$. The mixture was stirred at this temperature for 20 $\min$. Then, the soap stocks were separated by centrifugation at $2500 \mathrm{rpm}$.

Winterization: Without washing, $5 \%$ of $\mathrm{NaOH}\left(4^{\circ} \mathrm{Be}\right)$ was added to the neutralized oil, and the mixtures was kept at $5^{\circ} \mathrm{C}$ for $18 \mathrm{~h}$. The precipitates were separated by centrifugation.

Washes: In order to remove traces of dissolved soap, the winterized oil was washed three times with water $(10 \% \mathrm{w} / \mathrm{w})$ for $10 \mathrm{~min}$. The washing water was discarded.

Bleaching: The washed oil was dried under a vacuum of 60 Torr at $90^{\circ} \mathrm{C}$ for 15 min with an agitation of 125 rpm. Next, $1.5 \%$ of Tonsil Supreme $114 \%$ and $0.15 \%$ of activated carbon were added. The temperature was raised to $110^{\circ} \mathrm{C}$ and held for $10 \mathrm{~min}$. Then, the mixture was cooled under a vacuum and filtered at $50^{\circ} \mathrm{C}$.

Deodorization: The bleached oils were deodorized at $220^{\circ} \mathrm{C}$ for $3 \mathrm{~h}$ under a vacuum of 0.5 to $1 \mathrm{mbar}$. Once deodorized, the oils were filtered with filter paper and stored at $-18^{\circ} \mathrm{C}$ until analysis (OPOD CC and OPOD MAC).

\subsubsection{Physical refining process}

\subsubsection{Classical physical refining}

The steps of this procedure were as follows:

Degumming: Five hundred grams of OPOC MA was weighed into a vessel, and 0.5 wt $\%$ oil of citric acid was added to it. The mixture was maintained for $10 \mathrm{~min}$ at $80^{\circ} \mathrm{C}$ under agitation, to precipitate phospholipids in the acidic media.

Washes: In order to remove traces of dissolved soap, the degummed oil was washed once with water $(10 \% \mathrm{w} / \mathrm{w})$, for $10 \mathrm{~min}$ at $80^{\circ} \mathrm{C}$. The washing water was discarded.

Bleaching: The washed oil was dried under a vacuum of 60 Torr at $90^{\circ} \mathrm{C}$ for 15 min with an agitation of $125 \mathrm{rpm}$. Then, 1.5\% Tonsil Supreme 114, 0.1\% activated carbon, 1\% Trysil 300, and $0.1 \%$ Dicalite were added. The temperature was raised to $110^{\circ} \mathrm{C}$ and held for $10 \mathrm{~min}$. Then, the mixture was filtered. 
Neutralizing deodorization: The bleached oil was deodorized at $250^{\circ} \mathrm{C}$ (OPOD MAP) for 3 $\mathrm{h}$ under a vacuum of 0.5 to $1 \mathrm{mbar}$. Water vapor was used as the carrier gas at a rate of 6.24 $\mathrm{mL} / \mathrm{h}$. Once deodorized, the oil was filtered through paper and stored at $-18^{\circ} \mathrm{C}$ until analysis.

\subsubsection{Molecular distillation refining}

The steps of this procedure were as follows:

Bleaching: The bleaching conditions were the same as those used in the classical physical refining procedure.

Molecular distillation: The deodorization was performed in a short-path device (mod. KDL5, UIC Gmb, Germany). The operating conditions were fixed as follows: feed flow of 1.44 $\mathrm{l} / \mathrm{h}, 250 \mathrm{rpm}$ as the rotation speed of the rollers, condenser temperature of $50^{\circ} \mathrm{C}$, and vacuum of $0.001 \mathrm{mbar}$. Deodorized oils were taken at $140^{\circ} \mathrm{C}$ (OPOD140 MBP), $165^{\circ} \mathrm{C}$ (OPOD165 MBP), $190^{\circ} \mathrm{C}(\mathrm{OPOD} 190 \mathrm{MBP})$, and $215^{\circ} \mathrm{C}$ (OPOD215 MBP).

\subsection{Analytical study}

Analytical determination of bioactive compounds was carried out for crude olive pomace oils (OPOC C, OPOC MA, and OPOC MB) and deodorized oils obtained in different refining process (OPOD CC, OPOD MAC, OPOD MAP, and different OPOD MBP).

The acidity, fatty acid composition, wax content, and squalene and alkyl esters were determined by the methods outlined in the European Union [13].

The unsaponifiable matter was extracted and quantified after saponification of the oils with $\mathrm{KOH} / \mathrm{EtOH}$, following the Spanish standard method [14]. From these extracts, the content of sterols and aliphatic alcohols was determined according the proposed standard methods [13], by using 5 - $\alpha$-cholestan-3-ol and $n$-heneicosanol as internal standards, respectively.

The triterpenic acid content was determined following the method proposed by PérezCamino and Cert [2], which consists of the isolation of the fatty acid fraction by SPE-NH columns, silylation of the extracts, and quantification by gas chromatography (Mod. 7890A, Agilent Technologies), using betulinic acid as an internal standard.

\subsection{Rheological characterization}

Multi-step flow curve measurements were run using a controlled stress rheometer (AR2000, TA Instruments, New Castle, USA) and a plane geometry of 60-mm diameter with a 
smooth surface. A constant temperature was maintained with a Peltier plate $\left(25 \pm 0.1^{\circ} \mathrm{C}\right)$. The experimental protocol began with a dynamic oscillatory study to determine the linear viscoelastic region of oils through stress sweeps at a frequency of $1 \mathrm{~Hz}$, a percentage tolerance of $1 \%$, and maximum point time of $120 \mathrm{~s}$. Then, an appropriate value of stress was selected in order to comply with non-destructive conditions. After this, a frequency sweep was carried out in the range from $0.01 \mathrm{rad} / \mathrm{s}$ to $628.3 \mathrm{rad} / \mathrm{s}$. The analysis of results is based on the storage (G') and loss (G") moduli, which are related to the elastic and viscous components, respectively. On the other hand, flow and viscosity curves were determined by a rotational shear assay. Each measurement was made in triplicate and was deemed valid when the curves were superimposable.

Different theoretical models can be used to analyze the rheological results to test performance in terms of shear stress $(\tau)$ and shear rate $(\gamma)$ or viscosity $(\eta)$ and shear rate $(\gamma)$. The lowest standard error was applied to the theoretical rheological models. In our paper, the power law model (also well-known as Ostwald-de Waele model) was used Eq. (1).

$$
\tau=K \cdot \gamma^{n}
$$

This model relates the shear stress $(\tau)$ with the shear rate $(\gamma)$, where $K$ and $n$ are the consistency index and fluid index, respectively. Depending on the value of $n$, the fluid types can be determined. Fluids with $n=1$ are Newtonian fluids and those with $n \neq 1$ are nonNewtonian fluids. The flow behavior can be determined depending on the $n$ value. Thus, $n<1$ indicates pseudoplastic or plastic flow (shear-thinning) and $\mathrm{n}>1$ indicates dilatant flow (shearthickening).

\subsection{Texture profile analysis (TPA)}

The texture is defined by ISO 5492 [15] as: an instrumental technique that studies the set of rheological properties and structure of a perceived product by the mechano-receptors, touch receptors, and in some cases, by visuals and auditory information.

Actually, texture is an important attribute of any product, which affects its processing and handling, and determines its useful life as well as acceptance by users/patients.

Spreadability, in practical terms, is the ease with which a product can be spread in a thin, even layer on a surface. The firmness or hardness can be measured by the force required to obtain a given deformation or the amount of deformation by a given force. 
Although extensibility is also a deformation under an external load, it is a more dynamic property than firmness or hardness. Measurements of firmness and spreadability tend to be highly correlated, but this relationship is usually not perfect.

Textural properties of the different OPOCs and OPODs were determined by uniaxial compression with a load cell of $5 \mathrm{~kg}$. A texture analyzer TA.XT2i (Stable Micro Systems Ltd., Surrey, UK) was used and controlled by software. A conical probe (HDP/SR, Stable Micro Systems) was inserted in the complementary conical sample holder containing $8 \pm 0.1 \mathrm{~g}$ of different oils. This probe is an accessory that measures the ease with which a product can be applied in a thin, even layer. During the test, the force was increased to the point of maximum depth as the cone entered the vessel. The value of peak force (maximum force) was taken as the firmness to the specified depth. Firmer samples showed a larger positive area, representing the total amount of force (cohesiveness) required to perform the breaking process. Both values were used to quantify the spreadability of samples; however, one of the two measurements often gave more relevant information than the other. The adhesive properties of the product (adhesiveness), which are quantified by the negative zone of the curve, were characterized during the return movement of the probe [16].

\section{RESULTS AND DISCUSSION}

\subsection{Chemical characterization of OPOCs and OPODs obtained by refining processes.}

As mentioned before, OPOCs were refined to remove unwanted minor components from oils, while causing minimal losses of bioactive compounds [10]. Table 1 shows the results of analytical determinations of crude pomace oil obtained by: chemical (OPOC C) and mechanical (OPOC MA) extractions, and deodorized oil obtained by the chemical refining process (OPOD CC and OPOD MAC). Table 2 includes the corresponding results for oils obtained by mechanical extraction (OPOC MA and $O P O C M B$ ), and deodorized oils obtained by physical refining processes (OPOD MAP and OPOD MBP).

Acidity and triterpenic acids. In relation to the acidity, it is possible to appreciate two important aspects. First, it is noted that the oil obtained by mechanical extraction (OPOC MA and $O P O C M B$ ) has less acidity than that obtained by chemical extraction (OPOC C). Therefore, it seems that the centrifugation process is more suitable than the solvent extraction process. More nonpolar material was extracted in the first stage of the mechanical extraction, mainly triacylglycerols (TAG), whereas the later solvent stages contained more polar material as small amounts of other lipid components including non-esterified fatty acids, partial glycerides, 
long-chain alcohols and sterols. Besides, the chemical extraction process requires preliminary

255 drying under drastic conditions, producing oxidation and hydrolysis reactions and thus causing 256 the increase of free fatty acids.

Conversely it is observed that the temperature of the refining process greatly influences the acidity. Thus, it was observed that using high temperatures, as in the case of chemical $\left(220^{\circ} \mathrm{C}\right)$ and classical physical $\left(250^{\circ} \mathrm{C}\right)$ refining, acidity greatly decreases against the selected temperatures in molecular distillation. However, these high temperatures have a significant impact on the concentration of bioactive compounds.

In the case of chemical refining, free fatty acids and triterpenic acids were removed by saponification. The acidity was reduced below $<0.1 \%$; however, the oil quality was degraded from a health point of view because triterpenic acids had been removed. Similar behavior can be observed in the case of classical physical refining (Table 2). In contrast, the acidity was less reduced by molecular distillation, but a higher amount of anti-inflammatory bioactive compounds (triterpenic acids) were obtained (Table 2), because they cannot distill in the conditions used in the assays and contribute to the higher acidity values. This major acidity could be attributed to the fact that a steam stream was not introduced in the molecular distiller to remove free fatty acids.

Alkyl esters. As shown in Table 1 and 2, lower amounts of alkyl esters were obtained in all processes of refining. These non-bioactive compounds appear due to fermentation of vegetable matter during olive pomace oil storage in ponds [17]. Consistent with Yuan et al. [18], an increase in temperature causes a decrease of alkyl esters, as observed in the oils after molecular distillation, because these compounds are volatile at temperatures $>165^{\circ} \mathrm{C}$.

Waxes. These compounds are solids at room temperature. In general, the presence of long-chain esters as waxes in vegetable oil is used to determine the purity and classification of the various grades of olive oil [13]. The wax content increases during the chemical and physical refining processes (Tables 1 and 2). This suggests esterification reactions between free fatty acids and aliphatic alcohols present in the oil at deodorization temperatures, as reported by Tubaileh et al. [19]. Thus, when deodorization temperature increased, the wax content increased, as observed from the oils obtained by molecular distillation.

Unsaponifiable matter. This is composed of a complex mixture of compounds other than glycerides. The major bioactive compounds in olive pomace oil are squalene, sterols, and the triterpenic alcohols erythrodiol and uvaol [8]. As displayed in Table 1 and 2, the percentage of unsaponifiable matter decreased with refining. This could be attributed mainly to the removal of squalene in the refining processes. However, in molecular distillation, a significant amount 
of squalene was collected in deodorization distillates (OPOD140 MBP to OPOD215 MBP). This squalene, as indicated by Bondioli et al. [20], can be added to our oil, enriching the concentration of this bioactive compound.

Tables 1 and 2 report the different sterols characteristic of the olive pomace oil. In agreement with Verleyen et al. [21], the partial removal of sterols during the refining process depends largely on the conditions applied in the various stages of the process. Thus, with chemical and classical physical refining, the amount of these bioactive compounds decreased ( $40 \%$ and $20 \%$, respectively); in contrast, the amount of these sterols increased slightly in the molecular distillation. Similar behavior can be observed with triterpenic alcohols.

Considering all of the above, the physical refining processes, especially molecular distillation, contribute to better performance in terms of the bioactive compounds studied, which can be used in topical formulations for their therapeutical activities. Furthermore, of all the oils obtained by molecular distillation, OPOD190 MBP was chosen to be the only one that simultaneously shows low acidity and less loss of bioactive compounds.

In summary, the results obtained for OPOD190 MBP by molecular distillation were considered appropriate to compare with respect to EVOO, which is a raw material widely used in topical formulations (Table 3). It is noted that OPOD190 MBP showed greater acidity than EVOO, although still low; however, we confirmed that our olive pomace oil exhibited higher amounts of all bioactive compounds, except for squalene.

\subsection{Rheological characterization}

Fig. 2 shows the rheological behavior in chemical and mechanical extraction (OPOC C and OPOC MA), and chemical refining (OPOD CC and OPOD MAC). Fig. 2A presents the oscillatory frequency sweep for the measurement of viscoelasticity. In all cases, it was observed that the viscoelasticity of fluids, $\mathrm{G}^{\prime}$ and $\mathrm{G}^{\prime \prime}$, is dependent on the applied frequency. Moreover, there is a predominance of the viscous component in all OPOC as OPOD. $\mathrm{G}^{\prime}$ was only stable at frequencies less than 5-10 rad. $\mathrm{s}^{-1}$, indicating little influence of the elastic component in the viscoelastic behavior. At the same time, an important gap was observed in the profiles between $G^{\prime \prime}$ and G', which corroborates the impact of viscous behavior. Only OPOD CC shows little difference between the two components $\left(G^{\prime \prime}\right.$ and $\left.G^{\prime}\right)$, which were even reversed at frequencies below $0.1 \mathrm{rad} / \mathrm{s}$, indicating that $\mathrm{G}^{\prime}$ has greater impact on the internal structure of this oil.

Fig. 2B shows the flow curves of OPOC C, OPOC MA, OPOD CC, and OPOD MAC. All oils behave as pseudoplastic or shear-thinning fluids. 
Fig. 3 compares the rheological behavior in chemical and classical physical refining (OPOD MAC and OPOD MAP). For all oils, the viscous component ( $\left.G^{\prime \prime}\right)$ increased with the applied frequency (Fig. 3A). Therefore, OPOD MAC and OPOD MAP were viscoelastic fluids with a predominance of the viscous component, similar to OPOC MA. Furthermore, it was observed that OPOD MAP had progressively lower values of the elastic component ( $\left.G^{\prime}\right)$, in contrast with OPOD MAC.

The flow behavior can be observed in Fig. 3B, which shows that all oils were non-Newtonian fluids with pseudoplastic behavior.

Fig. 4 compares the rheological behavior of the two physical refining processes, classical physical (OPOD MAP) and molecular distillation (OPOD MBP). Again, all oils were viscoelastic fluids with a predominance of the viscous component, similar to raw olive pomace oils. Nonetheless, it is observed that the fractions subjected to temperatures above $145^{\circ} \mathrm{C}$ in the molecular distillation refining process (OPOD165 MBP, OPOD190 MBP and OPOD215 MBP) had progressively lower values of $\mathrm{G}^{\prime}$, and lost their elastic property (Fig. 4A).

The flow behavior can be observed in Fig. 4B. Again, all of the oils studied exhibited pseudoplastic flow behavior.

The flow curves were adjusted to different rheological theoretical models. The best fit was obtained with the power law model. Table 4 registers all the parameters obtained. The results confirm that all oils studied behave as Non-Newtonian fluids with pseudoplastic or shear-thinning behavior $(n<1)$. Nevertheless, the flow index values close to 1 denote that they were almost Newtonian fluids.

The consistency index $(\mathrm{K})$ values indicate that the viscosity was small in all instances. Moreover, the chemical refining process did not affect the flow characteristics (OPOD CC with respect to OPOC $C$ and OPOD MAC with respect to OPOC MA). Nonetheless, because OPOD CC and OPOD MAC did not differ in the refining process, the extraction process is responsible for the results obtained. Thus, mechanical extraction caused a loss of viscosity, while chemical extraction promoted an increase of it. This could be related with the profile of component extracted by means of solvent, included free fatty acids. It is important to mention that OPOC MA with lower acidity resulted in a decrease in viscosity. The opposite occurred in OPOC C, the sample with higher acidity (obtained by solvent extraction), increasing the viscosity of the sample [22].

The classical physical refining (OPOD MAP) displayed less viscosity than OPOC MA, although both are almost Newtonian fluids. The consistency index values were lower for the 
physically refined samples than for the crude olive pomace oil, while the chemical process seemed to not modify this parameter.

On the other hand, the molecular distillation refining process (OPOD MBP) slightly affected the values of the consistency index.

\subsection{Texture profile analysis (TPA)}

Determining the texture parameters of the oils can indicate their mechanical and sensory properties during situations of use, for example, spreadability, adhesiveness, and cohesiveness.

The firmness is related with the maximum positive force required to attain a given deformation. Cohesiveness and adhesiveness correspond to the areas under the curve obtained in the compression and withdrawal of the probe, respectively. Cohesiveness indicates the force to break the physical/chemical bonds established between the substances composing each oil. In contrast, adhesiveness indicates the force required to separate the oil from the walls of the containing recipient.

The results of TPA (Table 5) indicate that the refining processes influence these parameters. Thus, the chemical refining process shows that the deodorized oils (OPOD CC and OPOD MAC) present similar values. If the chemical and classical physical refining processes are compared, OPOD MAC and MAP OPOD show the lowest values of all parameters, indicating greater influence of classical physical refining than chemical refining. In the case of molecular distillation and taking into account all temperatures, changes in the texture values are less marked than in classical physical refining. As observed in Tables 1 and 2, waxes and triterpenic acids, solid compounds at room temperature, were higher in solvent extracted oils and presented higher contents after physical refining by molecular distillation.

In summary, OPOD CC and OPOD MAC are characterized by low firmness and cohesiveness, which indicate that they are soft and have high spreadability. At the same time, they have little adhesiveness and will thus form a thin film on the skin. OPOD MAP presents the same characteristics of texture.

Molecular distillation provides a battery of OPOD with optimal texture characteristics of softness and spreadability, which allow the formation of a more adhesive film on the skin. Therefore, these oils will constitute the raw materials in the design, already in development, of dermatological formulations. Although it will proceed to improve the characteristics of the oils with new techniques of refining. 


\section{CONCLUSIONS}

Temperature and, in general, the working conditions of the refining processes have important effects on the content of bioactive compounds. The physical refining processes, especially molecular distillation, contribute to better performance in these compounds. Furthermore, of all of the oils obtained by molecular distillation, OPOD190 MBP was chosen to be the only one that simultaneously showed low acidity and less loss of bioactive compounds. However, this oil showed higher acidity than extra virgin olive oil but higher amounts of all bioactive compounds, except squalene.

After the refining process, the olive pomace oils showed the best fit to the power law rheological model, possibly due to the removal of minor components in the process. In the OPOC subjected to refining, the viscosity decreased with an increase in the deodorization temperature and reduction in the amount of free fatty acids, thus also affecting the nature of the samples. The firmness, cohesiveness, and adhesiveness parameters from texture profile analysis showed that, in general, all OPODs have high smoothness and spreadability but low adhesiveness.

In view of the achieved results, it can be concluded that molecular distillation is the best refining process. Nevertheless, it is necessary to improve the working conditions in order to reduce acidity and achieve lower losses, if possible, of bioactive compounds. Therefore, other studies need to be performed by changing the working conditions of the molecular distillation in addition to applying other physical refining processes.

\section{ACKNOWLEDGMENTS}

The authors are grateful to Oleícola El Tejar, SCA for managing all the samples required for the development of this work and to Irene Pérez de la Rosa for technical assistance.

\section{REFERENCES}

[1] Draelos, Z.D., Cosmetic dermatology: products and procedures, John Wiley and Sons ed. Oxford. 2011

[2] Pérez-Camino, M.C., Cert, A., Quantitative determination of hydroxypentacyclic triterpene acids in vegetable oils. J. Agric. Food Chem. 1999, 47, 1558-1562.

[3] Rabionet, M., Gorgas, K., Sandhoff, R., Ceramide synthesis in the epidermis. BBA-Mol. Cell. Biol. L. 2014, 1841, 422-434. 
421 [4] Rabasco, A., González, M., Lipids in pharmaceutical and cosmetic preparations. Grasas $422 \quad$ Aceites. 2000, 51, 74-96.

423 [5] Sun, M., Tang, Y., Ding, T., Liu, M., Wang, X., Investigation of cytochrome P450 inhibitory 424 properties of maslinic acid, a bioactive compound from Olea europaea L., and its

[6] Reddy, L.H., Couvreur, P., Squalene: A natural triterpene for use in disease management and therapy. Adv. Drug. Deliver Rev. 2009, 61, 1412-1426.

[7] Fernández-Arche, A., Márquez-Martín, A., de la Puerta Vázquez, R., Perona, J., Terencio, C., Pérez-Camino, C., Ruiz-Gutiérrez, V., Long-chain fatty alcohols from pomace olive oil modulate the release of proinflammatory mediators. J. Nutr. Biochem. 2009, 20, 155-162.

[8] Márquez-Martín, A., De la Puerta, R., Fernández-Arche, A., Ruiz-Gutiérrez, V., Yaqoob, P., Modulation of cytokine secretion by pentacyclic triterpenes from olive pomace oil human mononuclear cells. Cytokine. 2006, 36, 211-217.

[9] Sánchez-Gutiérrez, C.A., Casas, M., Lucero, M.J., Ruiz-Méndez, M.V., Physico-chemical and rheological characterization of olive-pomace oils. Eur. J. Lipid. Sci. Tech. 2015, 117, 87-91.

[10] Ruiz-Méndez, M.V., Aguirre-González, M.R., Marmesat, S., Olive Oil Refining Process, in: Aparicio, R., Harwood, J. (Eds.), Handbook of Olive Oil. Springer US, 2013, pp. 715-738.

[11] Ruiz-Méndez, M.V., Dobarganes, M.C., Sánchez Moral, P., Edible olive pomace oil concentrated in triterpenic acids, procedure of physical refining utilized for obtainment thereof and recovery of functional components present in the crude oil. Patent ES 2332977 (A1) 2010.

[12] Amelotti, G., Effects of the interesterification for physical refining of some olive oils. Riv. Ital. Sostanze Grasse. 1987, 64, 223-226.

[13]Regulation (EU) No 1348/2013 amending Regulation (EEC) No 2568/91 on the characteristics of olive oil and olive-residue oil and on the relevant methods of analysis. Off. J. Eur. Union. L 338, 31-67.

[14] Standard Method UNE 55004. 2001. Quantitative Determination of Unsaponifiable Matter. AENOR (Asociación Española de Normalización y Racionalización). Madrid, Spain.

[15] UNE-EN ISO 5492:2010. Análisis sensorial. Vocabulario. (ISO 5492:2008). AENOR. Madrid.

[16] Aname Instrumentación Científica. http://analisisdetextura.blogspot.com.es/search /label/An\%C3\%A1lisis\%20de\%20Textura , 2016 (Accessed 30.05.16).

[17] Ruiz-Méndez, M.V., Ramos Hinojosa, A.E., Fatty acid esters with short chain alcohols in two phase olive pomace oils. Eur. J. Lipid. Sci. Tech. 2003, 105, 346-350. 
[18] Yuan, W., Hansen, A.C., Zhang, Q., Vapor pressure and normal boiling point predictions for pure methyl esters and biodiesel fuels. Fuel. 2005, 84, 943-950.

[19] Tubaileh, R.M., Garrido-Fernández, A., Ruiz Méndez, M.V., León-Camacho, M., et al., Effects of deodorization/physical refining variables in wax and fatty alcohols content of refined olive oil. J. Am. Oil Chem. Soc. 2002, 79, 67-71.

459

[20] Bondioli, P., Mariani, C., Lanzani, A., Fedeli, E., Muller, A., Squalene recovery from olive oil 460 deodorizer distillates. J. Am. Oil Chem. Soc. 1993, 70, 763-766.

461

[21] Verleyen, T., Sosinska, U., Verhé, R., Dewettinck, K., et al., Influence of the vegetable oil refining process on free and esterified sterols. J. Am. Oil Chem. Soc. 2002, 79, 947-953.

[22] Cedeño González, F.O., Prieto González, M.M., Bada Gancedo, J.C., Alonso Suárez, R., Estudio de la densidad y de la viscosidad de algunos ácidos grasos puros. Grasas Aceites. 1999, 50, 359-368.

466 